\section{LESSON 62}

Tabulation 21

PAPER: A5 $(210 \times 148 \mathrm{~mm})$.

TARGET TIME: 13 minutes.

Use the same techniques as in Lesson 61. Leave one space before and after each vertical line.

Tabulation 22

PAPER: A5 $(210 \times 148 \mathrm{~mm})$.

TARGET TIME: 15 minutes.

Tabulation 23

PAPER: A5 $(210 \times 148 \mathrm{~mm})$.

TARGET TIME: 15 minutes.

Rule up.

UNIT 16 \section{6}

\begin{tabular}{|l|l|l|}
\hline \multicolumn{1}{|c|}{ Gladioli } & \multicolumn{1}{|c|}{ Dahlia } & \multicolumn{1}{c|}{ Lily } \\
\hline Bloemfontein & $\begin{array}{l}\text { Gerrie Hoek } \\
\text { Scarlet Beauty }\end{array}$ & $\begin{array}{l}\text { Auratum } \\
\text { Enchantment } \\
\text { Fire King }\end{array}$ \\
High Summer & $\begin{array}{l}\text { Piquant } \\
\text { Prife Flame } \\
\text { Lovely Melody }\end{array}$ & $\begin{array}{l}\text { Poxy } \\
\text { Doyale }\end{array}$ \\
\hline
\end{tabular}

You will see that the two lines on the outside are missing. Carry on with the display as though you were going to rule up the outside lines: put in the underscore points for them, but do not rule them. Cover the underscore points when you rule the horizontal lines (those that run across the paper). Leave two spaces after the title - in other words, turn up three single lines to leave two blank.

\section{FOOTBALL CLUBS}

\begin{tabular}{l|l|l}
\hline \multicolumn{1}{c|}{ lst Division } & 2nd Division & 3rd Division \\
\hline $\begin{array}{l}\text { Arsenal } \\
\text { Derby } \\
\begin{array}{l}\text { Liveds } \\
\text { Manchester United }\end{array}\end{array}$ & $\begin{array}{l}\text { Carlisle United } \\
\text { Millwall } \\
\text { Oxford United } \\
\text { Preston }\end{array}$ & $\begin{array}{l}\text { Blackburn } \\
\text { Bolton } \\
\text { Bristol Rovers } \\
\text { Port Vale } \\
\text { Rotherham }\end{array}$ \\
\hline
\end{tabular}

PHOTOGRAPHIC EQUIPMENT

\begin{tabular}{|c|c|c|}
\hline Screens & Projectors & Enlargers \\
\hline $\begin{array}{l}\text { Alder Duomatic } \\
\text { Alder Square } \\
\text { Embassy } \\
\text { Viking Matador } \\
\text { Tracker Traveller } \\
\text { Tracker Starlight }\end{array}$ & $\begin{array}{l}\text { Adig } \mathrm{M} \\
\text { Adig } 0 \\
\text { Adig } \mathrm{Z} \\
\text { Arit Mig } \\
\text { Arit Super } \\
\text { Ican } 8\end{array}$ & $\begin{array}{l}\text { Black Knight } \\
\text { Migit } 5 \\
\text { Convoy } \\
\text { Adig Glow } \\
\text { Spread } 1 \\
\text { Maxi Minor }\end{array}$ \\
\hline
\end{tabular}

